\title{
Heavy Ion Testing at the Galactic Cosmic Ray Energy Peak
}

Jonathan A. Pellish, Michael A. Xapsos, Kenneth A. LaBel, Paul W. Marshall, David F. Heidel, Kenneth P. Rodbell, Mark C. Hakey, Paul E. Dodd, Marty R. Shaneyfelt, James R. Schwank, Robert C. Baumann, Xiaowei Deng, Andrew Marshall, Brian D. Sierawski, Jeffrey D. Black, Robert A. Reed, Ronald D. Schrimpf, Hak S. Kim, Melanie D. Berg, Michael J. Campola, Mark R. Friendlich, Christopher E. Perez, Anthony M. Phan, and Christina M. Seidleck

\section{5-word abstract}

A $1 \mathrm{GeV} / \mathrm{u}^{56} \mathrm{Fe}$ ion beam allows for true $90^{\circ}$ tilt irradiations of various microelectronic components and reveals relevant upset trends for an abundant element at the GCR flux energy peak.

\section{Corresponding Author:}

- Jonathan A. Pellish is with the Flight Data Systems and Radiation Effects Branch, NASA Goddard Space Flight Center, Code 561.4, 8800 Greenbelt RD, Greenbelt, MD 20771 USA. Email: jonathan.a.pellish@nasa.gov, Phone: +1 (301) 286-6523, Fax: +1 (301) 2864699

\section{Contributing Authors:}

- M. A. Xapsos and K. A. LaBel are with the Flight Data Systems and Radiation Effects Branch, NASA Goddard Space Flight Center, Code 561.4, 8800 Greenbelt RD, Greenbelt, MD 20771 USA.

- P. W. Marshall is a NASA consultant, Brookneal, VA 24528 USA.

- D. F. Heidel and K. P. Rodbell are with the IBM T. J. Watson Research Center, Yorktown Heights, NY 10598 USA.

- M. C. Hakey is with the IBM System and Technology Group, Essex Junction, VT 05452 USA.

- P. E. Dood, M. R. Shaneyfelt, and J. R. Schwank are with Sandia National Laboratories, Albuquerque, NM 87185 USA.

- R. C. Baumann, X. Deng, and A. Marshall are with Texas Instruments, Dallas, TX 75243 USA.

- B. D. Sierawski, J. D. Black, R. A. Reed, and R. D. Schrimpf are with the Department of Electrical Engineering and Computer Science, Vanderbilt University, Nashville, TN 37235 USA.

- H. S. Kim, M. D. Berg, M. J. Campola, M. R. Friendlich, C. E. Perez, A. M. Phan, and C. M. Seidleck are with MEI Technologies (NASA/GSFC), Greenbelt, MD 20771 USA.

Session Preference: Irradiation Facilities and Hardness Assurance Presentation Preference: Poster 


\title{
Heavy Ion Testing at the Galactic Cosmic Ray Energy Peak
}

\author{
Jonathan A. Pellish, Michael A. Xapsos, Kenneth A. LaBel, Paul W. Marshall, David F. Heidel, \\ Kenneth P. Rodbell, Mark C. Hakey, Paul E. Dodd, Marty R. Shaneyfelt, James R. Schwank, Robert C. Baumann, \\ Xiaowei Deng, Andrew Marshall, Brian D. Sierawski, Jeffrey D. Black, Robert A. Reed, Ronald D. Schrimpf, \\ Hak S. Kim, Melanie D. Berg, Michael J. Campola, Mark R. Friendlich, Christopher E. Perez, Anthony M. Phan, \\ and Christina M. Seidleck
}

\begin{abstract}
A $1 \mathrm{GeV} / \mathrm{u}^{56} \mathrm{Fe}$ ion beam allows for true $90^{\circ}$ tilt irradiations of various microelectronic components and reveals relevant upset trends for an abundant element at the GCR flux energy peak.
\end{abstract}

\section{INTRODUCTION}

A CCELERATED ground testing using heavy ions to study single-event effects (SEE) in microelectronic components differs from the actual space environment in two critical ways: one, ground-based accelerator heavy ion fluxes are much larger and two, ground-based accelerators cannot produce ions that cover the high-energy regime in space [1]. While these two issues do not prevent effective ground-based characterization of SEE, they tend to limit experimental conditions, some of which are important for hardness assurance. This paper describes recent heavy ion single-event upset (SEU) experiments at the NASA Space Radiation Effects Laboratory at Brookhaven National Laboratory using a ${ }^{56} \mathrm{Fe}$ beam with energies of $0.1,0.5$, and $1 \mathrm{GeV} / \mathrm{u}$. These energies correspond to linear energy transfers (LET) of 3.8, 1.5, and 1.2 $\left(\mathrm{MeV} \cdot \mathrm{cm}^{2}\right) / \mathrm{mg}$ in silicon. The devices under consideration include static random access memories (SRAM) and a field programmable gate array (FPGA).

Manuscript received 05 June 2009. This work was supported in part by the NASA Electronic Parts and Packaging program, the Space Radiation Element Human Research program at NASA/JSC, and the Defense Threat Reduction Agency Radiation Hardened Microelectronics Program under IACRO \#094587 I to NASA and \#09-4584I to SNL. Sandia is a multiprogram laboratory operated by Sandia Corporation, a Lockheed Martin Company, for the United States Department of Energy's National Nuclear Security Administration under Contract DE-AC04-94AL85000.

J. A. Pellish, M. A. Xapsos, and K. A. LaBel are with the Radiation Effects and Analysis Group, NASA/GSFC Code 561.4, 8800 Greenbelt RD, Greenbelt, MD 20771 USA. (email: jonathan.a.pellish@nasa.gov)

P. W. Marshall is a NASA consultant, Brookneal, VA 24528 USA.

D. F. Heidel and K. P. Rodbell are with the IBM T. J. Watson Research Center, Yorktown Heights, NY 10598 USA.

M. C. Hakey is with the IBM System and Technology Group, Essex Junction, VT 05452 USA.

P. E. Dood, M. R. Shaneyfelt, and J. R. Schwank are with Sandia National Laboratories, Albuquerque, NM 87185 USA.

R. C. Baumann, X. Deng, and A. Marshall are with Texas Instruments, Dallas, TX 75243 USA.

B. D. Sierawski, J. D. Black, R. A. Reed, and R. D. Schrimpf are with the Department of Electrical Engineering and Computer Science, Vanderbilt University, Nashville, TN 37235 USA.

H. S. Kim, M. D. Berg, M. J. Campola, M. R. Friendlich, C. E. Perez, A. M. Phan, and C. M. Seidleck are with MEI Technologies (NASA/GSFC), Greenbelt, MD 20771 USA.
Spacecraft must be designed to handle a number of different radiation environment hazards, including, but not limited to particle radiation, electromagnetic radiation, and orbital debris [2]. This work considers SEU hardness assurance for microelectronic components and thus focuses on the three categories of high-energy particle radiation in space. There are particles trapped in planetary magnetic fields, high fluxes of protons and heavy ions emitted from the sun during coronal mass ejections and solar flares, and a low flux, isotropic background of protons and heavy ions originating outside of the solar system called galactic cosmic rays (GCR). It is the third category, $\mathrm{GCR}$, that is relevant here.

GCR include all naturally occurring elements and have a flux energy peak of approximately $1 \mathrm{GeV} / \mathrm{u}$ [2]. GCR abundance is inversely proportional to atomic number with the exception of iron, which comprises a large amount of the total GCR flux beyond oxygen. These high-energy cosmic rays are very penetrating and have low LET values. While the GCR spectrum has higher LET components, they are much lower flux. Nevertheless, accelerated ground testing includes higher LET values to thoroughly characterize component response.

The maximum angle of irradiation in typical ground-based accelerator testing is governed by the device under test's (DUT) packaging and the range of the ion. While accelerated testing is often conducted at tilt angles between normal incidence and a maximum of perhaps $60-70^{\circ}$, half of the GCR flux is incident at angles greater than $60^{\circ}$. The solid angle of a cone, shown in Eq. 1, can be used to approximate a plane of sensitive devices. When the apex, $a$, is equal to $120^{\circ}, \Omega=\pi$, which is half the solid angle subtended by the surface of a hemisphere [3]. This means that half of the particles in an isotropic environment are incident at angles below $60^{\circ}$ and the other half at angles above $60^{\circ}$.

$$
\Omega=2 \pi\left[1-\cos \left(\frac{a}{2}\right)\right]
$$

Since a large number of heavy ions in the GCR spectrum are incident at grazing angles relative to the surface normal of the part, multiple-bit/cell upset (MBU) will be a significant concern [4]-[6]. MBUs are problematic because they can reduce or negate the effectiveness of error detection and correction codes [7]. This hardness assurance concern is further complicated by the fact that modern, highly-scaled process technologies $(\leq 100 \mathrm{~nm})$ are more sensitive to MBU [8]- 


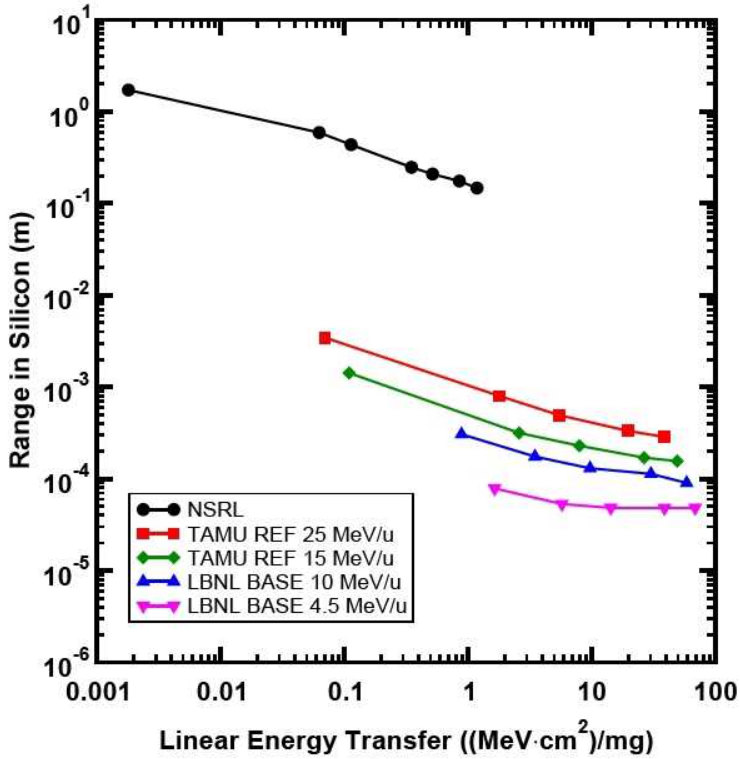

Fig. 1. The range vs. LET(Si) characterizations of two common heavy ion facilities relative to the NSRL. Ranges and LET values shown are for silicon. Note that the NSRL beams, at $1 \mathrm{GeV} / \mathrm{u}$, are representative of the flux energy peak of the GCR spectrum. The NSRL data points represent hydrogen, carbon, oxygen, silicon, chlorine, titanium, and iron. The TAMU REF data points come from helium, neon, argon, krypton, and xenon. The LBNL BASE data points come from boron, neon, argon, krypton, and xenon. All values are calculated at normal incidence.

[12]. This is the result of packing the sensitive nodes closer together and not necessarily an increase in upset sensitivity, particularly for technologies below $90 \mathrm{~nm}$. In several cases, the upset thresholds of these technologies are low enough to be affected by direct ionization from incident protons [13]-[15].

\section{EXPERIMENTAL FACILITY AND Setup}

The NASA Space Radiation Laboratory (NSRL) at Brookhaven National Laboratory (BNL) is a joint effort by the NASA Johnson Space Flight Center and the Department of Energy's Office of Science designed to study radiobiological effects relevant to human spaceflight. In addition to radiobiological studies, the NSRL also hosts physics experiments such as this work. Currently, heavy ions are accelerated using one of the two BNL Tandem van de Graaff accelerators and sent down a $700 \mathrm{~m}$ beamline to the Booster synchrotron. The beams are accelerated further in the Booster and then delivered to the NSRL. Because the Tandems serve as the ion source, the number of beams available at the NSRL is presently limited to hydrogen, carbon, oxygen, silicon, chlorine, titanium, and iron. However, with the commissioning of the electron beam ion source in 2010, all ions from hydrogen to uranium will be available and at much higher fluxes.

The beam itself is well-controlled and focused by two sets of magnetic lenses that can produce a "square" beam spot of up to $20 \mathrm{~cm} \times 20 \mathrm{~cm}$ with uniformity of $\pm 2 \%$; this was the beam used in this work. The staple energy tune at the NSRL is $1 \mathrm{GeV} / \mathrm{u}$, though the energy can be changed in about 10 minutes as long as the operators have advanced notice. The energy range is approximately $0.1 \mathrm{GeV} / \mathrm{u}$ to $1 \mathrm{GeV} / \mathrm{u}$, which is

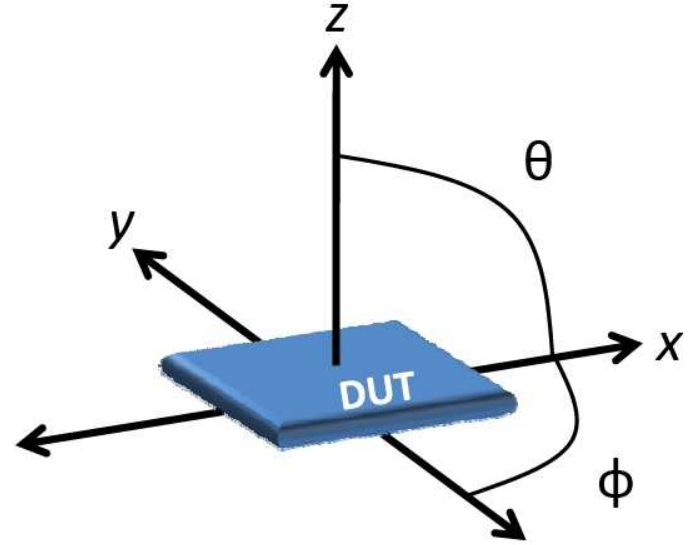

Fig. 2. The irradiation coordinate system is shown here. Exposures were conducted at normal incidence with a $-\hat{\mathbf{z}}$ beam vector, at $\left(\theta=90^{\circ}, \phi=0^{\circ}\right)$ with a $-\hat{\mathbf{x}}$ beam vector, and at $\left(\theta=90^{\circ}, \phi=90^{\circ}\right)$ with a $-\hat{\mathbf{y}}$ beam vector.

the energy at the DUT, not the extraction energy of the Booster synchrotron. At lower energies the beam is less uniform, with a small dip in intensity at the center of the beam spot. The ions are delivered to the target room in $300 \mathrm{~ms}$ spills approximately every $3.7 \mathrm{~s}$. Real-time dosimetry is achieved with a calibration ion chamber (a.k.a. EGG counter) manufactured by Far West Technologies in conjunction with larger secondary ion chambers. The secondary ion chambers are used to measure integrated dose and cut the beam off when a specific dose has been reached. The dosimetry unit is $\operatorname{rad}\left(\mathrm{H}_{2} \mathrm{O}\right)$ and must be converted to $\operatorname{rad}(\mathrm{Si})$ and then scaled by the LET of the incident beam in order to calculate the particle fluence.

In order to take advantage of the generous beam spot, jigs were made to hold four separate, coplanar DUTs - three SRAMs and one FPGA. For these experiments, the coordinate system is shown in Fig. 2, where $\theta$ is the tilt angle and $\phi$ is the roll angle. One jig is used for exposure at normal incidence and a separate jig is used for irradiation at a tilt angle of $90^{\circ}$. The latter jig is rolled $90^{\circ}$ about the $z$-axis.

\section{EXPERIMENTAL RESULTS}

\section{A. Static Random Access Memories}

Three SRAMs from two vendors, A and B, were exposed to the iron beam at the NSRL. Of the three, two are $65 \mathrm{~nm}$ and one is $45 \mathrm{~nm}$. One of the $65 \mathrm{~nm}$ SRAMs and the $45 \mathrm{~nm}$ SRAM are a silicon-on-insulator (SOI) process from vendor A; the remaining $65 \mathrm{~nm}$ part is a bulk complementary metal oxide semiconductor (CMOS) part from vendor B. Vendor A's $65 \mathrm{~nm}$ SOI SRAM is $1 \mathrm{Mbit}$ and their $45 \mathrm{~nm}$ SOI SRAM is 36 Mbit. Vendor B's bulk CMOS SRAM is 8 Mbit.

Vendor A's SEU and MBU cross sections for the 65 and $45 \mathrm{~nm}$ SOI SRAMs are shown in Fig. 3. The equations for the uncorrelated SEU and the correlated MBU cross sections are given by Eqs. 2 and 3 [6]. The SEU cross section is the total number of single-bit errors plus the multiplicity-corrected number of multi-bit errors divided by the uncorrected fluence - i.e., there are no RPP cosine corrections. The MBU cross section is the number of MBU events involving two or more physically adjacent bits divided by the uncorrected fluence. 


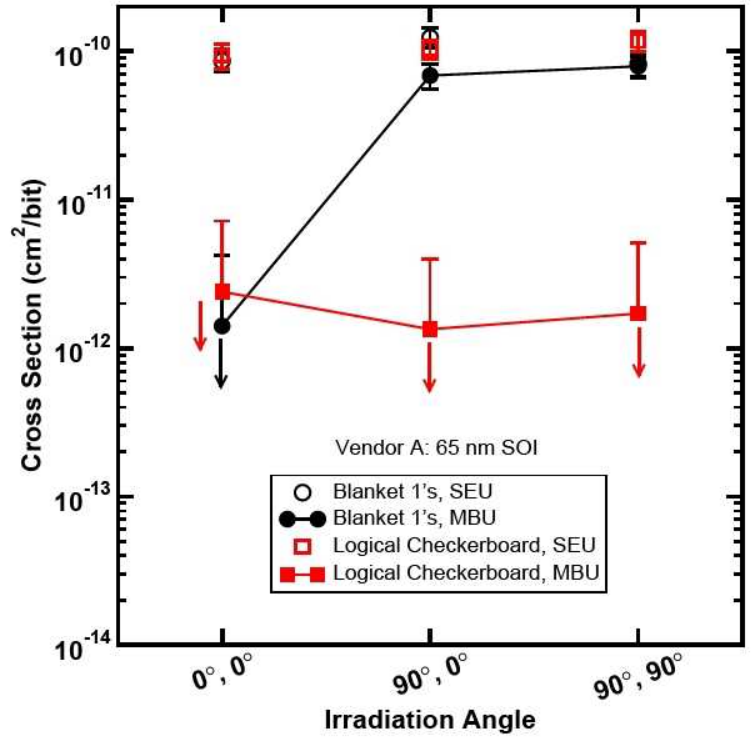

(a) Vendor A's $65 \mathrm{~nm}, 1 \mathrm{Mbit}$ SOI SRAM

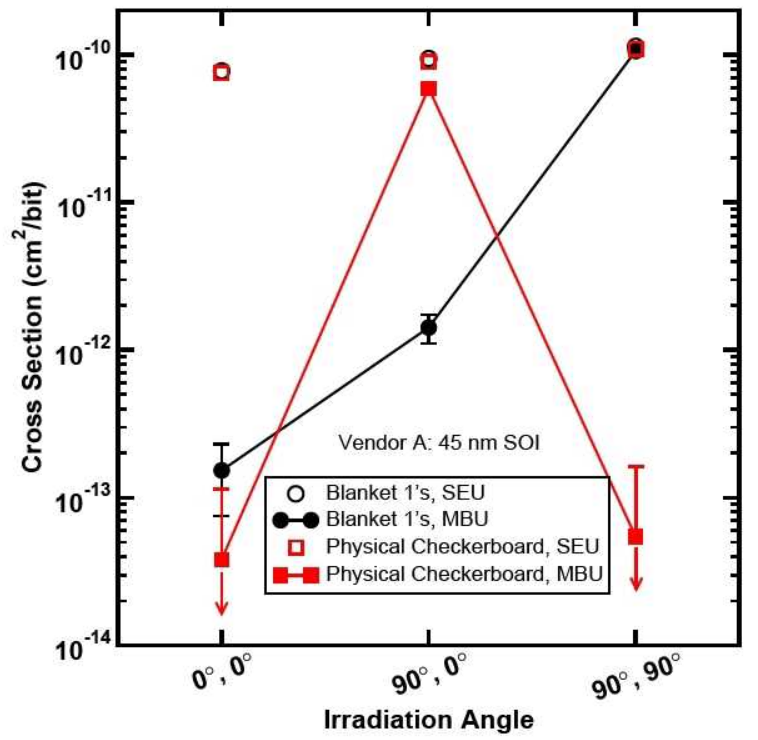

(b) Vendor A's $45 \mathrm{~nm}, 36$ Mbit SOI SRAM

Fig. 3. Vendor A's ${ }^{56} \mathrm{Fe}$ data. The abscissa categories are given as $(\theta, \phi)$ angle pairs; refer to Fig. 2 for a graphical representation. The legend shows the data pattern and the type of upset cross section. All irradiations used the $1 \mathrm{GeV} / \mathrm{u}^{56} \mathrm{Fe}$ beam. The $65 \mathrm{~nm}$ SOI SRAM in Fig. $3(\mathrm{a})$ was biased with $V_{\mathrm{DD}}=1.3 \mathrm{~V}$ and the $45 \mathrm{~nm}$ SOI SRAM in Fig. 3(b) was biased with $V_{\mathrm{DD}}=1.3 \mathrm{~V}$. Limiting cross sections are indicated by downward-pointing arrows.

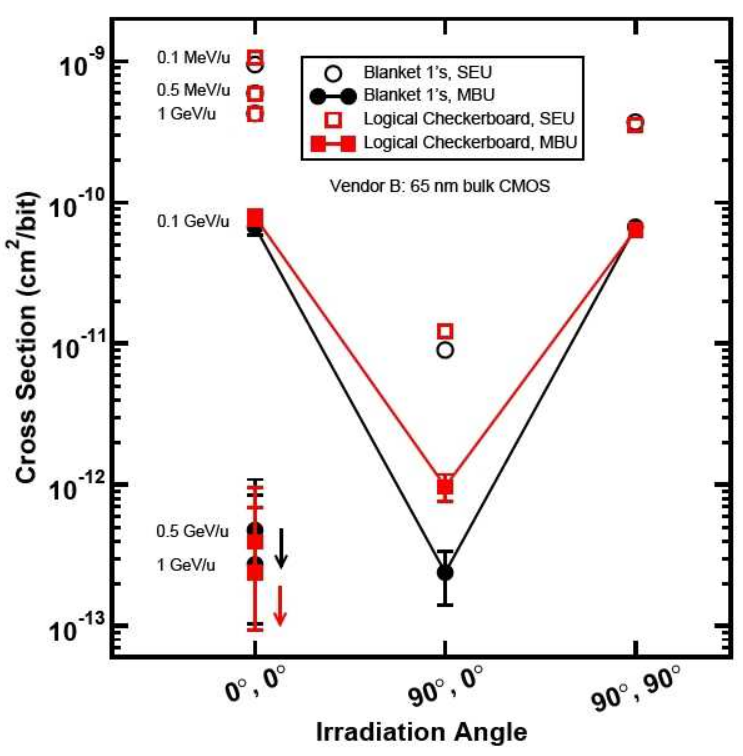

Fig. 4. Vendor B's $65 \mathrm{~nm}, 8$ Mbit bulk CMOS SRAM ${ }^{56} \mathrm{Fe}$ data. The abscissa categories are given as $(\theta, \phi)$ angle pairs; refer to Fig. 2 for a graphical representation. The legend shows the data pattern and the type of upset cross section. Irradiation energies are given at normal incidence since the data points are degenerate. The DUT was biased at $V_{\mathrm{DD}}=1.2 \mathrm{~V}$ for all exposures. Limiting cross sections are indicated by downward-pointing arrows.

Both the 65 and $45 \mathrm{~nm}$ SRAMs were irradiated at normal incidence, a tilt of $90^{\circ}$ and roll of $0^{\circ}$, and at a tilt of $90^{\circ}$ and a roll of $90^{\circ}$. All three of these irradiations were conducted with the $1 \mathrm{GeV} / \mathrm{u}{ }^{56} \mathrm{Fe}$ beam.

$$
\sigma_{\mathrm{SEU}}=\sum_{i=1}^{\infty} \frac{i \times \text { Event }_{i_{\mathrm{bit}}}}{\Phi}
$$

$$
\sigma_{\mathrm{MBU}}=\sum_{i=2}^{\infty} \frac{\text { Event }_{i_{\mathrm{bit}}}}{\Phi}
$$

Vendor B's SEU and MBU cross sections are shown in Fig. 4. This 8 Mbit bulk CMOS SRAM was exposed at 0.1, 0.5 , and $1 \mathrm{GeV} / \mathrm{u}$ at normal incidence and at $1 \mathrm{GeV} / \mathrm{u}$ for the other two orientations $-\left(90^{\circ}\right.$ tilt, $0^{\circ}$ roll $)$ and $\left(90^{\circ}\right.$ tilt, $90^{\circ}$ roll). Two data patterns were written to the memory - FF for blanket 1's and AA for a logical checkerboard. There is no significant difference in response for these two patterns. The data in Fig. 4 show a definite cross section dependence on grazing orientation with $\left(90^{\circ}\right.$ tilt, $90^{\circ}$ roll $)$ being the most sensitive. At this orientation both the SEU and MBU cross sections are larger than at the orthogonal roll angle with the same tilt. This indicates that the physical layout is responsible for the elevation in upset cross section. Physically adjacent MBUs as large as ten bits were observed at this orientation.

\section{B. Field Programmable Gate Array}

A $90 \mathrm{~nm}$ bulk CMOS, SRAM-based FPGA from Vendor C was exposed to the same beams as Vendor A's SRAM - 0.1, 0.5 , and $1 \mathrm{GeV} / \mathrm{u}{ }^{56} \mathrm{Fe}$ at normal incidence and $1 \mathrm{GeV} / \mathrm{u}{ }^{56} \mathrm{Fe}$ at $\left(90^{\circ}\right.$ tilt, $0^{\circ}$ roll $)$ and $\left(90^{\circ}\right.$ tilt, $90^{\circ}$ roll $)$. The results are shown in Fig. 5. A biased DUT was placed in the beam and the clocks were held static. The DUT underwent readback following exposure, recording the full contents of the configuration memory, which includes the logic configuration and block RAM (BRAM). The number of bits in error were calculated and then separated into configuration data and BRAM. This process was completed for two different, redundant FPGA designs - XTMR and DTMR. The cross section for each of these designs' configuration data and BRAM were calculated separately and are reported in Fig. 5. 


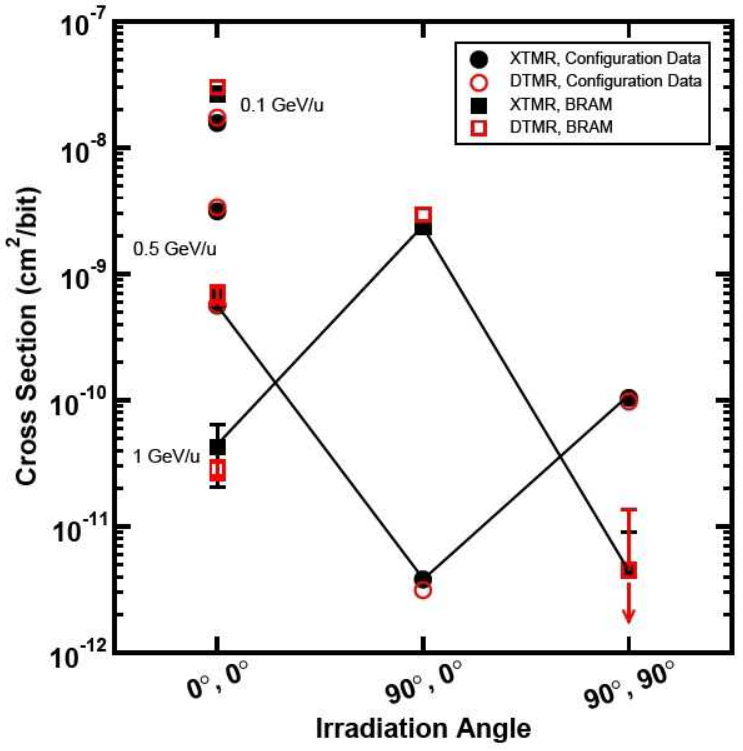

Fig. 5. Vendor C's $90 \mathrm{~nm}$ bulk CMOS FPGA ${ }^{56} \mathrm{Fe}$ static data. The abscissa categories are given as $(\theta, \phi)$ angle pairs; refer to Fig. 2 for a graphical representation. The legend shows the data pattern and the type of upset cross section. Irradiation energies are given at normal incidence since the data points are degenerate. The single limiting cross section is indicated by a downwardpointing arrow.

\section{DISCUSSION AND CONCLUSION}

The SRAM results are consistent with the fundamental differences between bulk CMOS and SOI technologies. The bulk technology has a thicker sensitive volume with many devices residing in the same $\mathrm{n}$ - and p-wells, making a large number of bits simultaneously susceptible to upset. This feature, while increasing the probability of high-multiplicity MBUs, also removes data pattern sensitivity since charge transport within the wells means that it is unnecessary for the incident ion to physically strike the necessary nodes to cause a cell state change.

The SOI SRAM data have a definite pattern and orientation dependence. Since each SRAM cell, and indeed some individual transistors within the SRAM cell, are isolated by oxide, the charge transport relevant in the bulk SRAM technology no longer applies. MBU in an SOI SRAM requires that the incident ion, or daughter particles in the case of indirect ionization, strike all the cells necessary to cause upset; charge transport plays almost no role.

The FPGA data, shown in Fig. 5, exhibits similar behavior to the SRAM data presented in Figs. 3 and 4, partly because the FPGA is SRAM-based, though the functionality of the two device types is very different. It is interesting to note that the configuration data and BRAM have opposing trends at $\left(90^{\circ}\right.$ tilt, $0^{\circ}$ roll $)$ and $\left(90^{\circ}\right.$ tilt, $90^{\circ}$ roll $)$, yielding information about the orientation of internal data storage.

This is the first time the NSRL facility has been used to irradiate highly-scaled commercial CMOS and SOI technologies. The $1 \mathrm{GeV} / \mathrm{u}{ }^{56} \mathrm{Fe}$ beam allowed true $90^{\circ}$ grazing angle irradiation of SRAM and FPGA parts without special die or package preparation. These experiments represent the current state-of-the-art for accelerated ground testing and allow for upset characterization with a realistic GCR heavy ion beam.

While it would be ideal to be able to execute these kind of experiments on a regular basis, experimental logistics and cost are significant barriers. The good news is that for most parts this kind to testing is unnecessary. Standard laboratory tilt angles will reveal the limiting case trends shown in Figs. 3, 4 , and 5 . However, these data also underscore the fact that data pattern and roll angle can and do play a significant role in upset cross section and thus are relevant concerns for single-event hardness assurance and must be investigated.

\section{ACKNOWLEDGMENT}

The authors would like to thank A. Rusek, M. Seiverts, P. Guida and the rest of the staff at the NSRL and the BNL Collider-Accelerator Department for excellent management and their not insignificant assistance in completing a successful test campaign at a new facility.

\section{REFERENCES}

[1] R. A. Reed et al., "Single-event effects ground testing and on-orbit rate prediction methods: the past, present, and future," IEEE Trans. Nucl. Sci., vol. 50, no. 3, pp. 622-634, Jun. 2003.

[2] M. A. Xapsos, "Modeling the space radiation environment," in Nuclear and Space Radiation Effects Conf. Short Course. Ponte Vedra Beach, FL: IEEE, 2006, pp. 1-62 Section II.

[3] J. A. Pellish et al., "A generalized SiGe HBT single-event effects model for on-orbit event rate calculations," IEEE Trans. Nucl. Sci., vol. 54, no. 6, pp. 2322-2329, Dec. 2007.

[4] R. A. Reed et al., "Heavy ion and proton-induced single event multiple upset," IEEE Trans. Nucl. Sci., vol. 44, no. 6, pp. 2224-2229, Dec. 1997.

[5] A. B. Campbell et al., "Analysis of single event effects at grazing angle [CMOS SRAMs]," IEEE Trans. Nucl. Sci., vol. 45, no. 3, pp. 16031611, Jun. 1998.

[6] A. D. Tipton et al., "Device-orientation effects on multiple-bit upset in 65 nm SRAMs," IEEE Trans. Nucl. Sci., vol. 55, no. 6, pp. 2880-2885, Dec. 2008.

[7] C. W. Slayman, "Cache and memory error detection, correction, and reduction techniques for terrestrial servers and workstations," IEEE Trans. Device Mater. Rel., vol. 5, no. 3, pp. 397-404, Sep. 2005.

[8] N. Seifert et al., "Radiation-induced soft error rates of advanced cmos bulk devices," in Int. Reliability Physics Symp., Mar. 2006, pp. 217-225.

[9] H. Quinn et al., "Domain crossing errors: Limitations on single device triple-modular redundancy circuits in Xilinx FPGAs," IEEE Trans. Nucl. Sci., vol. 54, no. 6, pp. 2037-2043, Dec. 2007.

[10] E. H. Cannon et al., "Multi-bit upsets in $65 \mathrm{~nm}$ SOI SRAMs," in Int. Reliability Physics Symp., May 2008, pp. 195-201.

[11] N. Seifert et al., "Multi-cell upset probabilities of $45 \mathrm{~nm}$ high-k + metal gate SRAM devices in terrestrial and space environments," in Int. Reliability Physics Symp., May 2008, pp. 181-186.

[12] H. Quinn et al., "An automated approach to estimating hardness assurance issues in triple-modular redundancy circuits in Xilinx FPGAs," IEEE Trans. Nucl. Sci., vol. 55, no. 6, pp. 3070-3076, Dec. 2008.

[13] K. P. Rodbell et al., "Low-energy proton-induced single-event-upsets in $65 \mathrm{~nm}$ node, silicon-on-insulator, latches and memory cells," IEEE Trans. Nucl. Sci., vol. 54 , no. 6, pp. 2474-2479, Dec. 2007.

[14] D. F. Heidel et al., "Low energy proton single-event-upset test results on $65 \mathrm{~nm}$ SOI SRAM," IEEE Trans. Nucl. Sci., vol. 55, no. 6, pp. 3394-3400, Dec. 2008.

[15] B. D. Siearwski et al., "Impact of low-energy proton induced upsets on test methods and rate predictions," in Nuclear and Space Radiation Effects Conf., 2009. 